Bull. Austral. Math. Soc.

26D15, 46C05

VOL. 69 (2004) [217-225]

\title{
ON THE BOAS-BELLMAN INEQUALITY IN \\ INNER PRODUCT SPACES
}

\section{S.S. DRAGOMIR}

New results related to the Boas-Bellman generalisation of Bessel's inequality in inner product spaces are given.

\section{INTRODUCTION}

Let $(H ;(\cdot, \cdot))$ be an inner product space over the real or complex number field $\mathbb{K}$. If $\left(e_{i}\right)_{1 \leqslant i \leqslant n}$ are orthonormal vectors in the inner product space $H$, that is, $\left(e_{i}, e_{j}\right)=\delta_{i j}$ for all $i, j \in\{1, \ldots, n\}$ where $\delta_{i j}$ is the Kronecker delta, then the following inequality is well known in the literature as Bessel's inequality (see for example [6, p. 391]):

$$
\sum_{i=1}^{n}\left|\left(x, e_{i}\right)\right|^{2} \leqslant\|x\|^{2} \text { for any } x \in H .
$$

For other results related to Bessel's inequality, see $[\mathbf{3}, \mathbf{4}, \mathbf{5}]$ and Chapter XV in the book [6].

In 1941, Boas [2] and in 1944, independently, Bellman [1] proved the following generalisation of Bessel's inequality (see also [6, p. 392]).

THEOREM 1. If $x, y_{1}, \ldots, y_{n}$ are elements of an inner product space $(H ;(\cdot, \cdot))$, then the following inequality:

$$
\sum_{i=1}^{n}\left|\left(x, y_{i}\right)\right|^{2} \leqslant\|x\|^{2}\left[\max _{1 \leqslant i \leqslant n}\left\|y_{i}\right\|^{2}+\left(\sum_{1 \leqslant i \neq j \leqslant n}\left|\left(y_{i}, y_{j}\right)\right|^{2}\right)^{1 / 2}\right]
$$

holds.

A recent generalisation of the Boas-Bellman result was given in Mitrinović-PečarićFink [6, p. 392] where they proved the following.

THEOREM 2. If $x, y_{1}, \ldots, y_{n}$ are as in Theorem 1 and $c_{1}, \ldots, c_{n} \in \mathbb{K}$, then one has the inequality:

$$
\left|\sum_{i=1}^{n} c_{i}\left(x, y_{i}\right)\right|^{2} \leqslant\|x\|^{2} \sum_{i=1}^{n}\left\|c_{i}\right\|^{2}\left[\max _{1 \leqslant i \leqslant n}\left\|y_{i}\right\|^{2}+\left(\sum_{1 \leqslant i \neq j \leqslant n}\left|\left(y_{i}, y_{j}\right)\right|^{2}\right)^{1 / 2}\right] .
$$

Received 30th June, 2003

Copyright Clearance Centre, Inc. Serial-fee code: 0004-9727/04 \$A2.00+0.00. 
They also noted that if in (1.3) one chooses $c_{i}=\overline{\left(x, y_{i}\right)}$, then this inequality becomes (1.2).

For other results related to the Boas-Bellman inequality, see [4].

In this paper we point out some new results that may be related to both the Mitrinović-Pečarić-Fink and Boas-Bellman inequalities.

\section{Some Preliminary Results}

We start with the following lemma which is also interesting in itself.

LEMmA 1. Let $z_{1}, \ldots, z_{n} \in H$ and $\alpha_{1}, \ldots, \alpha_{n} \in \mathbb{K}$. Then one has the inequality:

$$
\begin{aligned}
& \left\|\sum_{i=1}^{n} \alpha_{i} z_{i}\right\|^{2} \\
& \leqslant\left\{\begin{array}{l}
\max _{1 \leqslant i \leqslant n}\left|\alpha_{i}\right|^{2} \sum_{i=1}^{n}\left\|z_{i}\right\|^{2} \\
\left(\sum_{i=1}^{n}\left|\alpha_{i}\right|^{2 \alpha}\right)^{1 / \alpha}\left(\sum_{i=1}^{n}\left\|z_{i}\right\|^{2 \beta}\right)^{1 / \beta}, \quad \text { where } \alpha>1, \frac{1}{\alpha}+\frac{1}{\beta}=1 \\
\sum_{i=1}^{n}\left|\alpha_{i}\right|^{2} \max _{1 \leqslant i \leqslant n}\left\|z_{i}\right\|^{2}
\end{array}\right. \\
& +\left\{\begin{array}{l}
\max _{1 \leqslant i \neq j \leqslant n}\left\{\left|\alpha_{i} \alpha_{j}\right|\right\} \sum_{1 \leqslant i \neq j \leqslant n}\left|\left(z_{i}, z_{j}\right)\right| ; \\
{\left[\left(\sum_{i=1}^{n}\left|\alpha_{i}\right|^{\gamma}\right)^{2}-\left(\sum_{i=1}^{n}\left|\alpha_{i}\right|^{2 \gamma}\right)\right]^{1 / \gamma}\left(\sum_{1 \leqslant i \neq j \leqslant n}\left|\left(z_{i}, z_{j}\right)\right|^{\delta}\right)^{1 / \delta},} \\
\text { where } \gamma>1, \frac{1}{\gamma}+\frac{1}{\delta}=1 ; \\
{\left[\left(\sum_{i=1}^{n}\left|\alpha_{i}\right|\right)^{2}-\sum_{i=1}^{n}\left|\alpha_{i}\right|^{2}\right] \max _{1 \leqslant i \neq j \leqslant n}\left|\left(z_{i}, z_{j}\right)\right| .}
\end{array}\right.
\end{aligned}
$$

Proof: We observe that

$$
\begin{aligned}
\left\|\sum_{i=1}^{n} \alpha_{i} z_{i}\right\|^{2} & =\left(\sum_{i=1}^{n} \alpha_{i} z_{i}, \sum_{j=1}^{n} \alpha_{j} z_{j}\right) \\
& =\sum_{i=1}^{n} \sum_{j=1}^{n} \alpha_{i} \overline{\alpha_{j}}\left(z_{i}, z_{j}\right)=\left|\sum_{i=1}^{n} \sum_{j=1}^{n} \alpha_{i} \overline{\alpha_{j}}\left(z_{i}, z_{j}\right)\right| \\
& \leqslant \sum_{i=1}^{n} \sum_{j=1}^{n}\left|\alpha_{i}\right|\left|\overline{\alpha_{j}}\right|\left|\left(z_{i}, z_{j}\right)\right| \\
& =\sum_{i=1}^{n}\left|\alpha_{i}\right|^{2}\left\|z_{i}\right\|^{2}+\sum_{1 \leqslant i \neq j \leqslant n}\left|\alpha_{i}\right|\left|\alpha_{j}\right|\left|\left(z_{i}, z_{j}\right)\right| .
\end{aligned}
$$


Using Hölder's inequality, we may write that

$$
\begin{aligned}
& \sum_{i=1}^{n}\left|\alpha_{i}\right|^{2}\left\|z_{i}\right\|^{2} \\
& \leqslant\left\{\begin{array}{l}
\max _{1 \leqslant i \leqslant n}\left|\alpha_{i}\right|^{2} \sum_{i=1}^{n}\left\|z_{i}\right\|^{2} ; \\
\left(\sum_{i=1}^{n}\left|\alpha_{i}\right|^{2 \alpha}\right)^{1 / \alpha}\left(\sum_{i=1}^{n}\left\|z_{i}\right\|^{2 \beta}\right)^{1 / \beta}, \quad \text { where } \alpha>1, \frac{1}{\alpha}+\frac{1}{\beta}=1 ; \\
\sum_{i=1}^{n}\left|\alpha_{i}\right|^{2} \max _{1 \leqslant i \leqslant n}\left\|z_{i}\right\|^{2} .
\end{array}\right.
\end{aligned}
$$

By Hölder's inequality for double sums we also have

$$
\begin{aligned}
& \sum_{1 \leqslant i \neq j \leqslant n}\left|\alpha_{i}\right|\left|\alpha_{j}\right|\left|\left(z_{i}, z_{j}\right)\right| \\
& \leqslant\left\{\begin{array}{l}
\max _{1 \leqslant i \neq j \leqslant n}\left|\alpha_{i} \alpha_{j}\right| \sum_{1 \leqslant i \neq j \leqslant n}\left|\left(z_{i}, z_{j}\right)\right| ; \\
\left(\sum_{1 \leqslant i \neq j \leqslant n}\left|\alpha_{i}\right|^{\gamma}\left|\alpha_{j}\right|^{\gamma}\right)^{1 / \gamma}\left(\sum_{1 \leqslant i \neq j \leqslant n}\left|\left(z_{i}, z_{j}\right)\right|^{\delta}\right)^{1 / \delta}, \\
\text { where } \gamma>1, \frac{1}{\gamma}+\frac{1}{\delta}=1 ; \\
\sum_{1 \leqslant i \neq j \leqslant n}\left|\alpha_{i}\right|\left|\alpha_{j}\right| \max _{1 \leqslant i \neq j \leqslant n}\left|\left(z_{i}, z_{j}\right)\right|,
\end{array}\right. \\
& =\left\{\begin{array}{l}
\max _{1 \leqslant i \neq j \leqslant n}\left\{\left|\alpha_{i} \alpha_{j}\right|\right\} \sum_{1 \leqslant i \neq j \leqslant n}\left|\left(z_{i}, z_{j}\right)\right| ; \\
{\left[\left(\sum_{i=1}^{n}\left|\alpha_{i}\right|^{\gamma}\right)^{2}-\left(\sum_{i=1}^{n}\left|\alpha_{i}\right|^{2 \gamma}\right)\right]^{1 / \gamma}\left(\sum_{1 \leqslant i \neq j \leqslant n}\left|\left(z_{i}, z_{j}\right)\right|^{\delta}\right)^{1 / \delta},} \\
\text { where } \gamma>1, \frac{1}{\gamma}+\frac{1}{\delta}=1 ; \\
{\left[\left(\sum_{i=1}^{n}\left|\alpha_{i}\right|\right)^{2}-\sum_{i=1}^{n}\left|\alpha_{i}\right|^{2}\right] \max _{1 \leqslant i \neq j \leqslant n}\left|\left(z_{i}, z_{j}\right)\right| .}
\end{array}\right.
\end{aligned}
$$

Utilising (2.3) and (2.4) in (2.2), we may deduce the desired result (2.1).

REMARK 1. Inequality (2.1) contains in fact 9 different inequalities which may be obtained combining the first 3 ones with the last 3 ones.

A particular case that may be related to the Boas-Bellman result is embodied in the following inequality. 
COROLlaRY 1. With the assumptions in Lemma 1, we have

$$
\begin{aligned}
& \left\|\sum_{i=1}^{n} \alpha_{i} z_{i}\right\|^{2} \\
& \quad \leqslant \sum_{i=1}^{n}\left|\alpha_{i}\right|^{2}\left\{\max _{1 \leqslant i \leqslant n}\left\|z_{i}\right\|^{2}+\frac{\left[\left(\sum_{i=1}^{n}\left|\alpha_{i}\right|^{2}\right)^{2}-\sum_{i=1}^{n}\left|\alpha_{i}\right|^{4}\right]^{1 / 2}}{\sum_{i=1}^{n}\left|\alpha_{i}\right|^{2}}\left(\sum_{1 \leqslant i \neq j \leqslant n}\left|\left(z_{i}, z_{j}\right)\right|^{2}\right)^{1 / 2}\right\} \\
& \quad \leqslant \sum_{i=1}^{n}\left|\alpha_{i}\right|^{2}\left\{\max _{1 \leqslant i \leqslant n}\left\|z_{i}\right\|^{2}+\left(\sum_{1 \leqslant i \neq j \leqslant n}\left|\left(z_{i}, z_{j}\right)\right|^{2}\right)^{1 / 2}\right\}
\end{aligned}
$$

The first inequality follows by taking the third branch in the first curly bracket with the second branch in the second curly bracket for $\gamma=\delta=2$.

The second inequality in (2.5) follows by the fact that

$$
\left[\left(\sum_{i=1}^{n}\left|\alpha_{i}\right|^{2}\right)^{2}-\sum_{i=1}^{n}\left|\alpha_{i}\right|^{4}\right]^{1 / 2} \leqslant \sum_{i=1}^{n}\left|\alpha_{i}\right|^{2}
$$

Applying the following Cauchy-Bunyakovsky-Schwarz type inequality

$$
\left(\sum_{i=1}^{n} a_{i}\right)^{2} \leqslant n \sum_{i=1}^{n} a_{i}^{2}, \quad a_{i} \in \mathbb{R}_{+}, \quad 1 \leqslant i \leqslant n
$$

we may write that

$$
\left(\sum_{i=1}^{n}\left|\alpha_{i}\right|^{\gamma}\right)^{2}-\sum_{i=1}^{n}\left|\alpha_{i}\right|^{2 \gamma} \leqslant(n-1) \sum_{i=1}^{n}\left|\alpha_{i}\right|^{2 \gamma} \quad(n \geqslant 1)
$$

and

$$
\left(\sum_{i=1}^{n}\left|\alpha_{i}\right|\right)^{2}-\sum_{i=1}^{n}\left|\alpha_{i}\right|^{2} \leqslant(n-1) \sum_{i=1}^{n}\left|\alpha_{i}\right|^{2} \quad(n \geqslant 1)
$$

Also, it is obvious that:

$$
\max _{1 \leqslant i \neq j \leqslant n}\left\{\left|\alpha_{i} \alpha_{j}\right|\right\} \leqslant \max _{1 \leqslant i \leqslant n}\left|\alpha_{i}\right|^{2}
$$

Consequently, we may state the following coarser upper bounds for $\left\|\sum_{i=1}^{n} \alpha_{i} z_{i}\right\|^{2}$ that may be useful in applications. 
COROLlary 2. With the assumptions in Lemma 1, we have the inequalities:

$$
\begin{aligned}
& \left\|\sum_{i=1}^{n} \alpha_{i} z_{i}\right\|^{2} \\
& \leqslant\left\{\begin{array}{l}
\max _{1 \leqslant i \leqslant n}\left|\alpha_{i}\right|^{2} \sum_{i=1}^{n}\left\|z_{i}\right\|^{2} \\
\left(\sum_{i=1}^{n}\left|\alpha_{i}\right|^{2 \alpha}\right)^{1 / \alpha}\left(\sum_{i=1}^{n}\left\|z_{i}\right\|^{2 \beta}\right)^{1 / \beta}, \text { where } \alpha>1, \frac{1}{\alpha}+\frac{1}{\beta}=1 \\
\sum_{i=1}^{n}\left|\alpha_{i}\right|^{2} \max _{1 \leqslant i \leqslant n}\left\|z_{i}\right\|^{2}
\end{array}\right. \\
& +\left\{\begin{array}{l}
\max _{1 \leqslant i \leqslant n}\left|\alpha_{i}\right|^{2} \sum_{1 \leqslant i \neq j \leqslant n}\left|\left(z_{i}, z_{j}\right)\right| ; \\
(n-1)^{1 / \gamma}\left(\sum_{i=1}^{n}\left|\alpha_{i}\right|^{2 \gamma}\right)^{1 / \gamma}\left(\sum_{1 \leqslant i \neq j \leqslant n}\left|\left(z_{i}, z_{j}\right)\right|^{\delta}\right)^{1 / \delta}, \\
\text { where } \gamma>1, \frac{1}{\gamma}+\frac{1}{\delta}=1 ; \\
(n-1) \sum_{i=1}^{n}\left|\alpha_{i}\right|^{2} \max _{1 \leqslant i \neq j \leqslant n}\left|\left(z_{i}, z_{j}\right)\right| .
\end{array}\right.
\end{aligned}
$$

The proof is obvious by Lemma 1 in applying the inequalities (2.7)-(2.9).

REMARK 2. The following inequalities which are incorporated in (2.10) are of special interest:

$$
\begin{aligned}
\left\|\sum_{i=1}^{n} \alpha_{i} z_{i}\right\|^{2} \leqslant \max _{1 \leqslant i \leqslant n}\left|\alpha_{i}\right|^{2}\left[\sum_{i=1}^{n}\left\|z_{i}\right\|^{2}\right. & \left.+\sum_{1 \leqslant i \neq j \leqslant n}\left|\left(z_{i}, z_{j}\right)\right|\right] \\
\left\|\sum_{i=1}^{n} \alpha_{i} z_{i}\right\|^{2} \leqslant\left(\sum_{i=1}^{n}\left|\alpha_{i}\right|^{2 p}\right)^{1 / p}\left[\left(\sum_{i=1}^{n}\left\|z_{i}\right\|^{2 q}\right)^{1 / q}\right. & \left.+(n-1)^{1 / p}\left(\sum_{1 \leqslant i \neq j \leqslant n}\left|\left(z_{i}, z_{j}\right)\right|^{q}\right)^{1 / q}\right],
\end{aligned}
$$

where $p>1,1 / p+1 / q=1$; and

$$
\left\|\sum_{i=1}^{n} \alpha_{i} z_{i}\right\|^{2} \leqslant \sum_{i=1}^{n}\left|\alpha_{i}\right|^{2}\left[\max _{1 \leqslant i \leqslant n}\left\|z_{i}\right\|^{2}+(n-1) \max _{1 \leqslant i \neq j \leqslant n}\left|\left(z_{i}, z_{j}\right)\right|\right] .
$$

\section{Some Mitrinović-Pećarić-Fink TyPe Inequalities}

We are now able to point out the following result which complements the inequality (1.3) due to Mitrinović, Pečarić and Fink [6, p. 392].

THEOREM 3 . Let $x, y_{1}, \ldots, y_{n}$ be vectors of an inner product space $(H ;(\cdot, \cdot))$ and 
$c_{1}, \ldots, c_{n} \in \mathbb{K}(\mathbb{K}=\mathbb{C}, \mathbb{R})$. Then one has the inequalities:

(3.1) $\left|\sum_{i=1}^{n} c_{i}\left(x, y_{i}\right)\right|^{2}$

$$
\begin{aligned}
& \leqslant\|x\|^{2} \times\left\{\begin{array}{l}
\max _{1 \leqslant i \leqslant n}\left|c_{i}\right|^{2} \sum_{i=1}^{n}\left\|y_{i}\right\|^{2} ; \\
\left(\sum_{i=1}^{n}\left|c_{i}\right|^{2 \alpha}\right)^{1 / \alpha}\left(\sum_{i=1}^{n}\left\|y_{i}\right\|^{2 \beta}\right)^{1 / \beta}, \quad \text { where } \alpha>1, \frac{1}{\alpha}+\frac{1}{\beta}=1 ; \\
\sum_{i=1}^{n}\left|c_{i}\right|^{2} \max _{1 \leqslant i \leqslant n}\left\|y_{i}\right\|^{2},
\end{array}\right. \\
& +\|x\|^{2} \times\left\{\begin{array}{l}
\max _{1 \leqslant i \neq j \leqslant n}\left\{\left|c_{i} c_{j}\right|\right\} \sum_{1 \leqslant i \neq j \leqslant n}\left|\left(y_{i}, y_{j}\right)\right| ; \\
{\left[\left(\sum_{i=1}^{n}\left|c_{i}\right|^{\gamma}\right)^{2}-\left(\sum_{i=1}^{n}\left|c_{i}\right|^{2 \gamma}\right)\right]^{1 / \gamma}\left(\sum_{1 \leqslant i \neq j \leqslant n}\left|\left(y_{i}, y_{j}\right)\right|^{\delta}\right)^{1 / \delta}} \\
{\left[\left(\sum_{i=1}^{n}\left|c_{i}\right|\right)^{2}-\sum_{i=1}^{n}\left|c_{i}\right|^{2}\right]}
\end{array}\right.
\end{aligned}
$$

PROOF: We note that

$$
\sum_{i=1}^{n} c_{i}\left(x, y_{i}\right)=\left(x, \sum_{i=1}^{n} \overline{c_{i}} y_{i}\right)
$$

Using Schwarz's inequality in inner product spaces, we have:

$$
\left|\sum_{i=1}^{n} c_{i}\left(x, y_{i}\right)\right|^{2} \leqslant\|x\|^{2}\left\|\sum_{i=1}^{n} \overline{c_{i}} y_{i}\right\|^{2} .
$$

Now using Lemma 1 with $\alpha_{i}=\overline{c_{i}}, z_{i}=y_{i}(i=1, \ldots, n)$, we deduce the desired inequality (3.2).

The following particular inequalities that may be obtained by the Corollaries 1 and 2 and Remark 2 hold. 
COROLlaRY 3. With the assumptions in Theorem 3, one has the inequalities:

$$
\begin{aligned}
& \left|\sum_{i=1}^{n} c_{i}\left(x, y_{i}\right)\right|^{2} \\
& \leqslant \times\left\{\begin{array}{l}
\|x\|^{2} \sum_{i=1}^{n}\left|c_{i}\right|^{2}\left\{\max _{1 \leqslant i \leqslant n}\left\|y_{i}\right\|^{2}+\left(\sum_{1 \leqslant i \neq j \leqslant n}\left|\left(y_{i}, y_{j}\right)\right|^{2}\right)^{1 / 2}\right\} \\
\|x\|^{2} \max _{1 \leqslant i \leqslant n}\left|c_{i}\right|^{2}\left\{\sum_{i=1}^{n}\left\|y_{i}\right\|^{2}+\sum_{1 \leqslant i \neq j \leqslant n}\left|\left(y_{i}, y_{j}\right)\right|\right\} \\
\|x\|^{2}\left(\sum_{i=1}^{n}\left|c_{i}\right|^{2 p}\right)^{1 / p}\left\{\left(\sum_{i=1}^{n}\left\|y_{i}\right\|^{2 q}\right)^{1 / q}+(n-1)^{1 / p}\left(\sum_{1 \leqslant i \neq j \leqslant n}\left|\left(y_{i}, y_{j}\right)\right|^{q}\right)^{1 / q}\right\}, \\
\text { where } p>1, \frac{1}{p}+\frac{1}{q}=1 ; \\
\|x\|^{2} \sum_{i=1}^{n}\left|c_{i}\right|^{2}\left\{\max _{1 \leqslant i \leqslant n}\left\|y_{i}\right\|^{2}+(n-1) \max _{1 \leqslant i \neq j \leqslant n}\left|\left(y_{i}, y_{j}\right)\right|\right\} .
\end{array}\right.
\end{aligned}
$$

REMARK 3. Note that the first inequality in (3.2) is the result obtained by MitrinovićPečarić-Fink in [6]. The other 3 provide similar bounds in terms of the $p$-norms of the vector $\left(\left|c_{1}\right|^{2}, \ldots,\left|c_{n}\right|^{2}\right)$.

\section{Some Boas-Bellman Type Inequalities}

If one chooses $c_{i}=\overline{\left(x, y_{i}\right)}(i=1, \ldots, n)$ in $(3.2)$, then it is possible to obtain 9 different inequalities between the Fourier coefficients $\left(x, y_{i}\right)$ and the norms and inner products of the vectors $y_{i}(i=1, \ldots, n)$. We restrict ourselves only to those inequalities that may be obtained from (3.2).

As Mitrinović, Pečarić and Fink noted in [6, p. 392], the first inequality in (3.2) for the above selection of $c_{i}$ will produce the Boas-Bellman inequality (1.2).

From the second inequality in (3.2) for $c_{i}=\overline{\left(x, y_{i}\right)}$ we get

$$
\left(\sum_{i=1}^{n}\left|\left(x, y_{i}\right)\right|^{2}\right)^{2} \leqslant\|x\|^{2} \max _{1 \leqslant i \leqslant n}\left|\left(x, y_{i}\right)\right|^{2}\left\{\sum_{i=1}^{n}\left\|y_{i}\right\|^{2}+\sum_{1 \leqslant i \neq j \leqslant n}\left|\left(y_{i}, y_{j}\right)\right|\right\} \text {. }
$$

Taking the square root in this inequality we obtain:

$$
\sum_{i=1}^{n}\left|\left(x, y_{i}\right)\right|^{2} \leqslant\|x\| \max _{1 \leqslant i \leqslant n}\left|\left(x, y_{i}\right)\right|\left\{\sum_{i=1}^{n}\left\|y_{i}\right\|^{2}+\sum_{1 \leqslant i \neq j \leqslant n}\left|\left(y_{i}, y_{j}\right)\right|\right\}^{1 / 2},
$$

for any $x, y_{1}, \ldots, y_{n}$ vectors in the inner product space $(H ;(\cdot, \cdot))$.

If we assume that $\left(e_{i}\right)_{1 \leqslant i \leqslant n}$ is an orthonormal family in $H$, then by (4.1) we have

$$
\sum_{i=1}^{n}\left|\left(x, e_{i}\right)\right|^{2} \leqslant \sqrt{n}\|x\| \max _{1 \leqslant i \leqslant n}\left|\left(x, e_{i}\right)\right|, \quad x \in H .
$$


From the third inequality in (3.2) for $c_{i}=\overline{\left(x, y_{i}\right)}$ we deduce

$$
\begin{aligned}
\left(\sum_{i=1}^{n}\left|\left(x, y_{i}\right)\right|^{2}\right)^{2} \leqslant\|x\|^{2}( & \left.\sum_{i=1}^{n}\left|\left(x, y_{i}\right)\right|^{2 p}\right)^{1 / p} \\
& \times\left\{\left(\sum_{i=1}^{n}\left\|y_{i}\right\|^{2 q}\right)^{1 / q}+(n-1)^{1 / p}\left(\sum_{1 \leqslant i \neq j \leqslant n}\left|\left(y_{i}, y_{j}\right)\right|^{q}\right)^{1 / q}\right\},
\end{aligned}
$$

for $p>1,1 / p+1 / q=1$.

Taking the square root in this inequality we get

$$
\begin{aligned}
\sum_{i=1}^{n}\left|\left(x, y_{i}\right)\right|^{2} \leqslant\|x\| & \left(\sum_{i=1}^{n}\left|\left(x, y_{i}\right)\right|^{2 p}\right)^{1 / 2 p} \\
& \times\left\{\left(\sum_{i=1}^{n}\left\|y_{i}\right\|^{2 q}\right)^{1 / q}+(n-1)^{1 / p}\left(\sum_{1 \leqslant i \neq j \leqslant n}\left|\left(y_{i}, y_{j}\right)\right|^{q}\right)^{1 / q}\right\}^{1 / 2},
\end{aligned}
$$

for any $x, y_{1}, \ldots, y_{n} \in H, p>1,1 / p+1 / q=1$.

The above inequality (4.3) becomes, for an orthornormal family $\left(e_{i}\right)_{1 \leqslant i \leqslant n}$,

$$
\sum_{i=1}^{n}\left|\left(x, e_{i}\right)\right|^{2} \leqslant n^{1 / q}\|x\|\left(\sum_{i=1}^{n}\left|\left(x, e_{i}\right)\right|^{2 p}\right)^{1 / 2 p}, \quad x \in H .
$$

Finally, the choice $c_{i}=\overline{\left(x, y_{i}\right)}(i=1, \ldots, n)$ will produce in the last inequality in (3.2)

$$
\left(\sum_{i=1}^{n}\left|\left(x, y_{i}\right)\right|^{2}\right)^{2} \leqslant\|x\|^{2} \sum_{i=1}^{n}\left|\left(x, y_{i}\right)\right|^{2}\left\{\max _{1 \leqslant i \leqslant n}\left\|y_{i}\right\|^{2}+(n-1) \max _{1 \leqslant i \neq j \leqslant n}\left|\left(y_{i}, y_{j}\right)\right|\right\}
$$

giving the following Boas-Bellman type inequality

$$
\sum_{i=1}^{n}\left|\left(x, y_{i}\right)\right|^{2} \leqslant\|x\|^{2}\left\{\max _{1 \leqslant i \leqslant n}\left\|y_{i}\right\|^{2}+(n-1) \max _{1 \leqslant i \neq j \leqslant n}\left|\left(y_{i}, y_{j}\right)\right|\right\}
$$

for any $x, y_{1}, \ldots, y_{n} \in H$.

It is obvious that (4.5) will give for orthonormal families the well known Bessel inequality.

REMARK 4. In order the compare the Boas-Bellman result with our result (4.5), it is enough to compare the quantities

$$
A:=\left(\sum_{1 \leqslant i \neq j \leqslant n}\left|\left(y_{i}, y_{j}\right)\right|^{2}\right)^{1 / 2}
$$

and

$$
B:=(n-1) \max _{1 \leqslant i \neq j \leqslant n}\left|\left(y_{i}, y_{j}\right)\right|
$$


Consider the inner product space $H=\mathbb{R}$ with $(x, y)=x y$, and choose $n=3, y_{1}=a>0$, $y_{2}=b>0, y_{3}=c>0$. Then

$$
A=\sqrt{2}\left(a^{2} b^{2}+b^{2} c^{2}+c^{2} a^{2}\right)^{1 / 2}, \quad B=2 \max (a b, a c, b c) .
$$

Denote $a b=p, b c=q, c a=r$. Then

$$
A=\sqrt{2}\left(p^{2}+q^{2}+r^{2}\right)^{1 / 2}, \quad B=2 \max (p, q, r) .
$$

Firstly, if we assume that $p=q=r$, then $A=\sqrt{6} p, B=2 p$ which shows that $A>B$.

Now choose $r=1$ and $p, q=1 / 2$. Then $A=\sqrt{3}$ and $B=2$ showing that $B>A$.

Consequently, in general, the Boas-Bellman inequality and our inequality (4.5) cannot be compared.

\section{REFERENCES}

[1] R. Bellman, 'Almost orthogonal series', Bull. Amer. Math. Soc. 50 (1944), 517-519.

[2] R.P. Boas, 'A general moment problem', Amer. J. Math. 63 (1941), 361-370.

[3] S.S. Dragomir and J. Sándor, 'On Bessel's and Gram's inequality in prehilbertian spaces', Period. Math. Hungar. 29 (1994), 197-205.

[4] S.S. Dragomir and B. Mond, 'On the Boas-Bellman generalisation of Bessel's inequality in inner product spaces', Ital. J. Pure Appl. Math. 3 (1998), 29-35.

[5] S.S. Dragomir, B. Mond and J.E. Pečarić, 'Some remarks on Bessel's inequality in inner product spaces', Studia Univ. Babes-Bolyai Math. 37 (1992), 77-86.

[6] D.S. Mitrinović, J.E. Pečarić and A.M. Fink, Classical and new inequalities in analysis (Kluwer Academic Publishers, Dordrecht, 1993).

School of Computer Science and Mathematics

Victoria University of Technology

PO Box 14428

MCMC, Vic 8001

Australia

e-mail: sever.dragomir@vu.edu.au

urladdr: http://rgmia.vu.edu.au/SSDragomirWeb.html 